\title{
ESTABILIDADE DO ÁCIDO ASCÓRBICO EM SUCO DE LARANJA (Citrus sinensis) IN NATURA, EM SUCO REFRIGERADO E EM PREPARADOS QUENTES
}

\section{STABILITY OF THE ASCORBIC ACID IN ORANGE (Citrus sinensis) JUICE IN NATURA, COOLED JUICE AND HOT CHEMICAL PREPARATIONS}

\author{
Ana Paula de Faria Alves ${ }^{1}$; Janine V. Santos ${ }^{2}$; Laudelina B. Neves ${ }^{3}$; Maria Josiane Sereia ${ }^{4}$ \\ 1,2,3,4 Universidade Tecnológica Federal do Paraná - UTFPR - Campo Mourão - Brasil. \\ 10aulafarialves@gmail.com; ${ }^{4}$ mjsereia@yahoo.com.br
}

\section{Resumo}

Foi realizada avaliação comparativa da retenção da vitamina C (ácido Ascórbico - AA) em suco fresco de laranjas íntegras, suco de laranja estocada sob refrigeração e em bolo contendo suco de laranja após a cocção em forno culinário convencional. O teor de AA em suco de laranjas armazenadas sob refrigeração por oito semanas variou entre 74,94 e 51,55 mg\% com taxa de redução de 0,92\% ao dia. Em suco fresco de laranja armazenado sob refrigeração durante sete dias, o teor médio de AA foi de 49,62 mg\%, com taxa de redução de aproximadamente 0,89\% ao dia do teor de AA. A retenção de AA foi 97,91\% nas laranjas íntegras e no suco refrigerado por sete dias 50,38\% A redução de AA observado nas preparações a quente foi de aproximadamente 73\%, sendo necessário, neste caso, compensar as perdas deste nutriente no planejamento dietético.

Palavras chave: ácido ascórbico; suco de laranja; processamento térmico.

\section{Introdução}

A grande disponibilidade e aceitabilidade do suco de laranja fazem com que muitos consumidores o considerem a melhor fonte de ácido ascórbico (AA) de sua dieta, embora existam alimentos mais ricos neste nutriente (RUSCHEL et al., 2001; SHAW et al., 1991). Embora o teor de AA varie significativamente entre diferentes cultivares de laranja, um volume médio de $200 \mathrm{~mL}$ de suco, dos cultivares "Pêra”, "Seleta”, "Lima” e "Lima da Pérsia” são suficientes para suprir as recomendações diárias desta vitamina (VALENTE-MESQUITA, 2002).

Extrai-se da laranja uma bebida única rica em vitamina $C$ e outros componentes que têm papel importante na prevenção de doenças, onde copo de suco desta fruta é o suficiente para que o organismo humano receba a quantidade diária recomendada de vitamina C (GAYET, 1999).

A vitamina C é essencial à saúde, desempenha papel fundamental no desenvolvimento e regeneração dos músculos, pele, dentes e ossos, na formação do colágeno, na regulação da 
temperatura corporal, na produção de diversos hormônios e no metabolismo em geral. A falta dessa vitamina no organismo aumenta a propensão a doenças. A carência severa torna o organismo vulnerável a doenças mais graves, como por exemplo, o escorbuto. Entretanto, consumida em altas doses, pode provocar efeitos colaterais, tais como: diarréia, dor abdominal e cálculos renais em pessoas geneticamente predispostas. A necessidade diária de vitamina C varia conforme idade e condições de saúde. Assim, as frutas frescas, principalmente as cítricas, são fontes ideais de vitamina C. O tomate, folhas verdes, que contem teores variáveis dessa vitamina e outras frutas como acerola, caju, goiaba e uva, são fontes alternativas de vitamina C (DAVIS et al., 1991; DOSUNMU e JOHNSON, 1995; GARDENER et al., 2001; WILLIANS, 1997).

Enfim, estudos bioquímicos relacionados ao AA abordam aspectos imunológicos, oncológicos, endocrinológicos, neurológicos, digestivos. Estudos de absorção e prevenção da catarata também foram realizados (DAVIS et al., 1991).

Trabalho realizado em 1955 por Dhopeshwarkar e Margar, citados por Camargo et al., (1984), estudaram o efeito do processamento térmico e do armazenamento em diversas frutas e hortaliças e verificaram que a perda do ácido ascórbico em produtos assim conservados é quase total se armazenados em temperaturas acima de $37^{\circ} \mathrm{C}$.

De acordo com Kordich (1997), o suco de frutas cítricas deve ser consumido imediatamente após o preparo, para melhor aproveitamento da vitamina C. Também é recomendado que sejam incluídas as películas e a membrana para obtenção dos benefícios de suas vitaminas e bioflavonóides.

Embora, de modo geral, a estabilidade da vitamina C aumente com o abaixamento da temperatura e a maior perda se dá durante o aquecimento dos alimentos, existem casos de perda durante o congelamento ou armazenamento a baixas temperaturas (BOBBIO; BOBBIO, 1995).

Segundo Fennema (1993), o ácido ascórbico é muito sensível a diversas formas de degradação, entre numerosos fatores que podem influir nos mecanismos degradativos caber citar a temperatura, tempo a concentração de sal e açúcar, o pH, o oxigênio, as enzimas, os catalisadores metálicos, a concentração inicial do ácido e a relação ácido ascórbico/ácido dehidroascórbico, bem como traços de cobre e ferro, sendo sua conservação é favorecida em meio ácido.

De acordo com Camargo et al (1984), recomenda, para melhor conservação da vitamina nos alimentos, o armazenamento em baixa temperatura, rápido pré-aquecimento (para destruir as enzimas oxidantes), além do mínimo contato com o oxigênio atmosférico. Segundo eles, a pasteurização, o cozimento, a desidratação e a evaporação destroem parcialmente a vitamina C, devido a sua alta solubilidade. Relatam ainda que o suco de citros e de tomate enlatados ou congelados contém os mesmos teores de vitamina $\mathrm{C}$ das frutas in natura. 
O presente trabalho teve como objetivo avaliar a taxa de redução do teor de acido ascórbico em suco de laranjas íntegras estocadas sob refrigeração por oito semanas, em suco de laranja in natura estocado sob refrigeração, e retenção de ácido ascórbico em suco de laranja utilizado como único ingrediente fonte de vitamina $\mathrm{C}$ em preparos a quente (bolo).

\section{Material e Métodos}

\subsection{Amostras}

As análises foram realizadas no Laboratório de Análise de Alimentos da Universidade Tecnológica Federal do Paraná Campus de Campo Mourão, durante os meses de novembro de 2005 a Janeiro de 2006.

As laranjas da variedade "pêra” (Citrus sinensis) foram adquiridas em mercado varejista da cidade de Campo Mourão e transportadas para o laboratório de análise e processamento de alimentos da Universidade Tecnológica Federal do Paraná, higienizadas e acondicionada sob refrigeração $\left(4^{\circ} \mathrm{C}\right)$ para a realização das análises por oito semanas.

Foi extraído manualmente o suco de laranja, filtrado e realizadas as análises de:

a) Determinação do acido ascórbico no suco de laranja no momento zero, com um dia, dois dias e sete dias;

b) Determinação do ácido ascórbico no bolo pós assamento;

c) Determinação a acidez titulável total (ATT) no momento zero, com um dia, dois dias e sete dias;

d) Determinação do ${ }^{\circ}$ Brix (sólidos solúveis totais) no momento zero, com um dia, dois dias e sete dias.

\subsection{Métodos}

\subsubsection{Análises físico-químicas}

As análises físico-quimícas foram realizadas de acordo com a metodologia oficial descrita pelo Instituto Adolfo Lutz (INSTITUTO ADOLFO LUTZ, 1995).

a) O teor de ácido ascórbico (AA), expresso em mg de AA/100 mL de suco foi determinado, em triplicata, através do método do Iodato de Potássio (método de Balentine);

b) A acidez total titulável (ATT), foi determinada, em triplicata, por titulação com $\mathrm{NaOH} \mathrm{0,1}$ $\mathrm{eq} / \mathrm{L}$;

c) Determinou-se o pH das amostras em pHmetro digital da marca Labmeter calibrado com soluções tampão de pH 4,0 e pH 7,0.

d) $\mathrm{O}^{\circ}$ Brix foi realizada por leitura em refratômetro de bancada a $20^{\circ} \mathrm{C}$. 


\subsubsection{Análise estatística}

Os resultados obtidos foram submetidos à análise de variância ANOVA no programa Software SPSS 10,0.

\section{Resultados e discussão}

O Ministério da Agricultura por meio da Instrução Normativa n ${ }^{0} 1$ de 7 de janeiro de 2000, determina os padrões para suco de laranja industrializado, onde deve apresentar teores mínimos de AA e de sólidos solúveis totais (SST), de 25,00 mg\% e de $10,5{ }^{\circ}$ Brix, respectivamente, além de uma relação SST/acidez total titulável (ATT), em g/100g de ácido cítrico anidro, mínimo de 7,0.

\section{1 Ácido Ascórbico}

Para os parâmetros físico-químicos analisados, as amostras não diferiram estatisticamente entre si, conforme a Tabela 1.

Tabela 1 - Valores das médias dos parâmetros físico-químicos analisados do suco de laranjas armazenadas sob refrigeração durante oito semanas.

\begin{tabular}{ccccc}
\hline & AA & Acidez & pH & ${ }^{\circ}$ Brix \\
\hline Momento & Média $(\mathrm{mg} \%)$ & Média & Média & Média \\
\hline $1^{\text {a }}$ semana & $74,94^{\mathrm{a}}$ & $7,40^{\mathrm{a}}$ & $4,30^{\mathrm{a}}$ & $9,81^{\mathrm{a}}$ \\
$2^{\mathrm{a}}$ semana & $77,14^{\mathrm{a}}$ & $6,92^{\mathrm{a}}$ & $4,50^{\mathrm{a}}$ & $10,06^{\mathrm{a}}$ \\
$3^{\mathrm{a}}$ semana & $71,11^{\mathrm{a}}$ & $7,74^{\mathrm{a}}$ & $4,39^{\mathrm{a}}$ & $11,78^{\mathrm{a}}$ \\
$4^{\mathrm{a}}$ semana & $71,24^{\mathrm{a}}$ & $7,93^{\mathrm{a}}$ & $4,35^{\mathrm{a}}$ & $12,15^{\mathrm{a}}$ \\
$5^{\mathrm{a}}$ semana & $69,36^{\mathrm{a}}$ & $7,74^{\mathrm{a}}$ & $4,47^{\mathrm{a}}$ & $11,65^{\mathrm{a}}$ \\
$6^{\mathrm{a}}$ semana & $60,76^{\mathrm{a}}$ & $8,02^{\mathrm{a}}$ & $4,19^{\mathrm{a}}$ & $11,90^{\mathrm{a}}$ \\
$7^{\mathrm{a}}$ semana & $55,59^{\mathrm{a}}$ & $8,03^{\mathrm{a}}$ & $4,23^{\mathrm{a}}$ & $10,88^{\mathrm{a}}$ \\
$8^{\mathrm{a}}$ semana & $51,55^{\mathrm{a}}$ & $7,41^{\mathrm{a}}$ & $4,52^{\mathrm{a}}$ & $10,88^{\mathrm{a}}$ \\
\hline
\end{tabular}

Letras iguais na mesma coluna não diferem estatisticamente entre si.

Para Fennema (1993), o ácido ascórbico é muito sensível, fato este observado na comparação a sua perda na Tabela 2. A média do teor de ácido ascórbico no tempo zero foi maior comparados entre os três tempos (24, 48 e 168h) e os parâmetros físico-químicos como pH, acidez e ${ }^{\circ}$ Brix não diferiram estatisticamente entre si.

Tabela 2 - Valores das médias dos parâmetros físico-químicos analisados do suco de laranjas armazenado sob refrigeração entre zero e 168 horas.

\begin{tabular}{ccccc}
\hline & AA & Acidez & $\mathrm{pH}$ & ${ }^{\circ}$ Brix \\
\hline Momento & Média (mg\%) & Média & Média & Média \\
\hline $0 \mathrm{~h}$ & $97,91^{\mathrm{a}}$ & $7,20^{\mathrm{a}}$ & $4,34^{\mathrm{a}}$ & $11,01^{\mathrm{a}}$ \\
$24 \mathrm{~h}$ & $62,84^{b}$ & $7,68^{\mathrm{a}}$ & $4,36^{\mathrm{a}}$ & $11,28^{\mathrm{a}}$ \\
$48 \mathrm{~h}$ & $55,46^{b}$ & $8,01^{\mathrm{a}}$ & $4,31^{\mathrm{a}}$ & $10,94^{\mathrm{a}}$ \\
$168 \mathrm{~h}$ & $49,62^{b}$ & $7,70^{\mathrm{a}}$ & $4,46^{\mathrm{a}}$ & $11,32^{\mathrm{a}}$ \\
\hline
\end{tabular}

\footnotetext{
Letras diferentes na mesma coluna diferem estatisticamente entre si.
} 
A figura 1 apresenta a representação gráfica da variação do ácido ascórbico (AA) no tempo zero com variação de 57\%. O teor de AA em suco de laranjas armazenadas durante oito semanas variou entre 74,94 a 51,55 mg\% diminuindo com o tempo de armazenamento.

Figura 1 - Variação do AA em horas durante um período de oito semanas

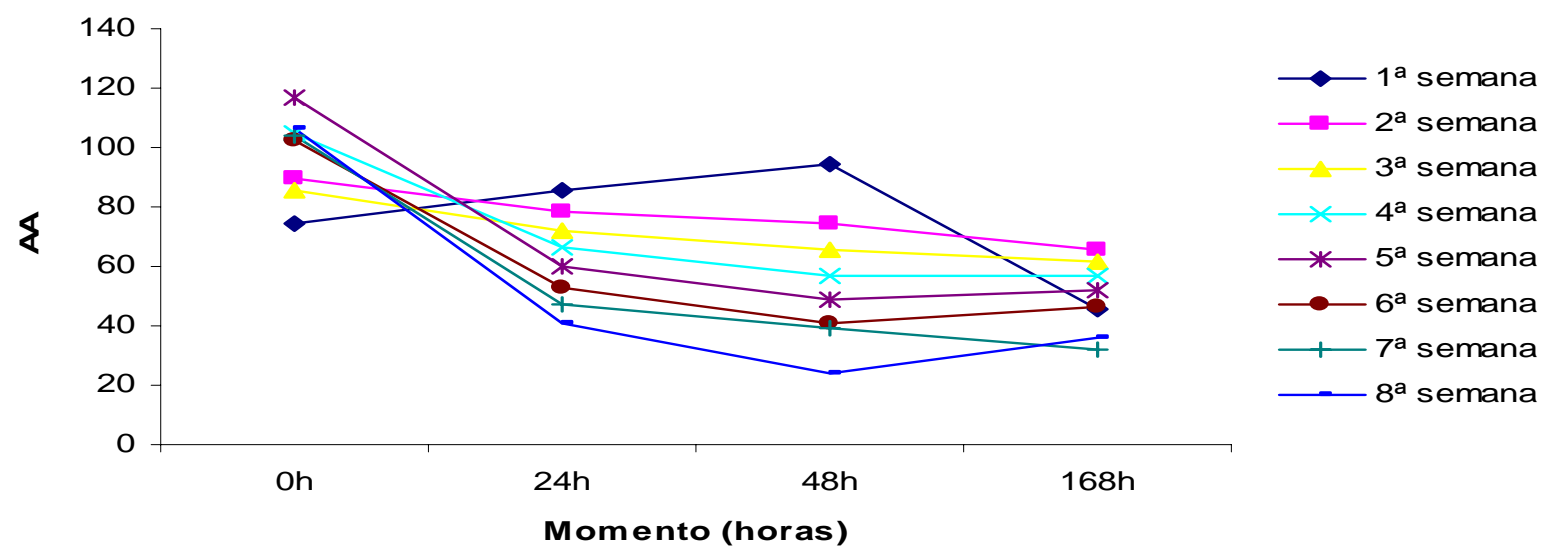

O período de 168 horas apresentou uma variação em relação aos períodos anteriores (24h e 48h). Em relação há zero hora, provavelmente os picos observados em algumas semanas, se deve ao maior grau de amadurecimento das laranjas que constituíram estes lotes. Estas variações estão apresentadas na Figura 2.

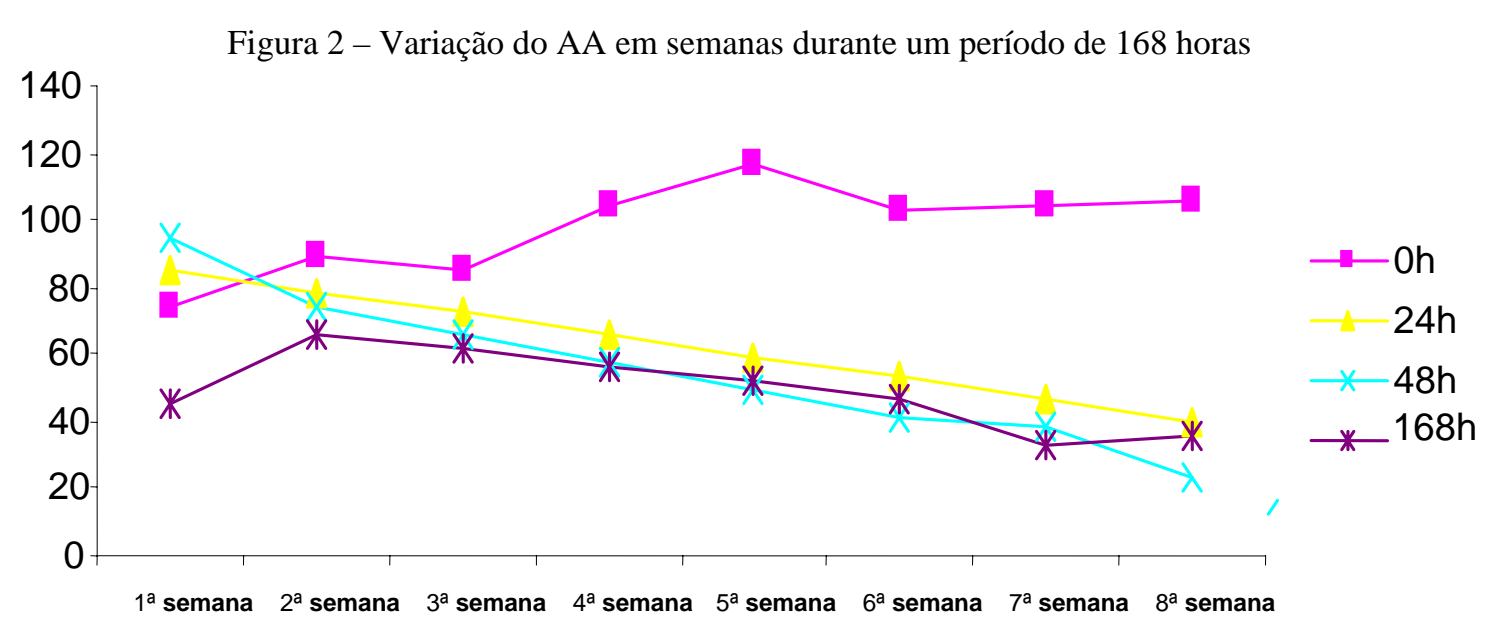

A Figura 3 apresenta o teor de AA no bolo após cocção, onde se apresentou valores diferentes na $1^{\text {a }}$ semana (23,5 $\mathrm{mg} \%$ ) e na $4^{\text {a }}$ semana (29,7 $\mathrm{mg} \%$ ), com média de 26,36 mg\% e desvio padrão de 2,18, com pequena variabilidade dos dados (coeficiente de variação de 8\%). Estas variações devem ao fato dos diferentes pontos de maturação das frutas. 
Figura 3 - Variação do AA em semanas no bolo

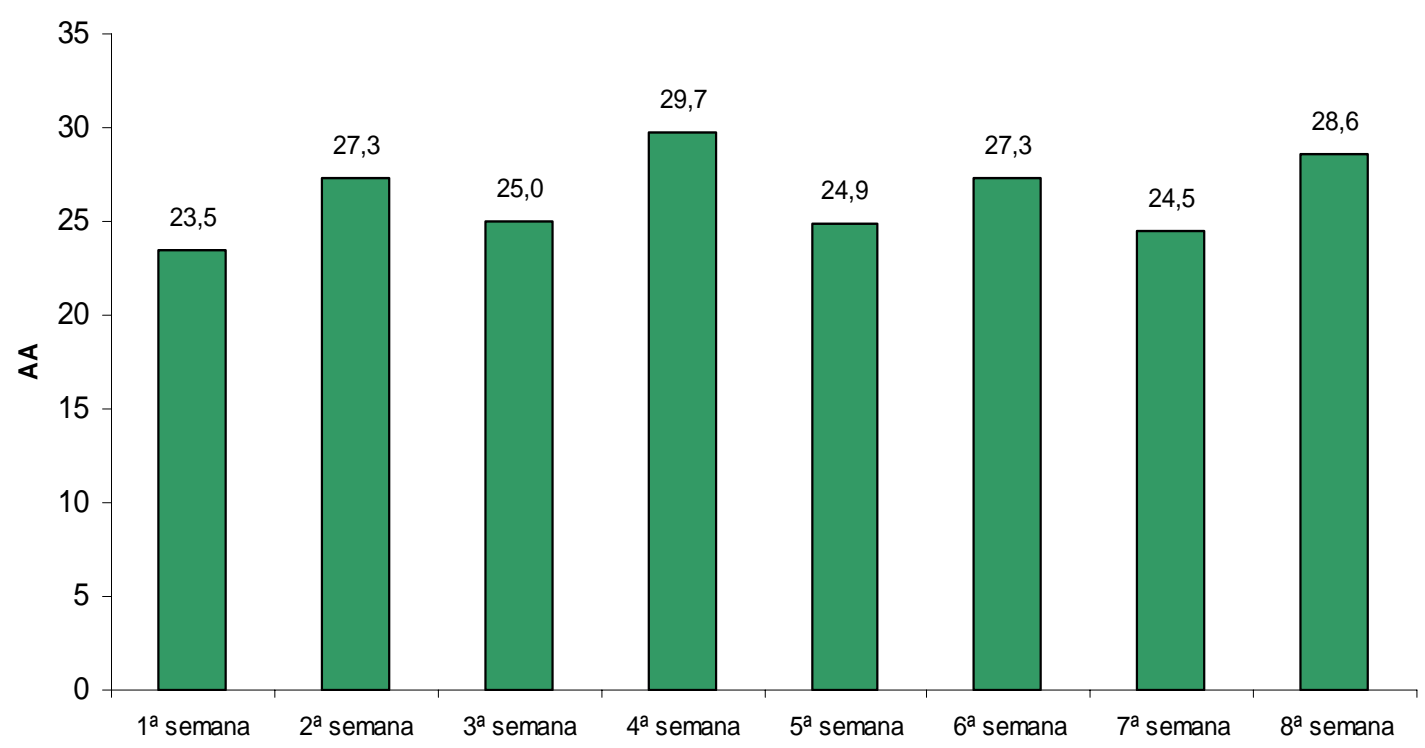

Os resultados das análises de AA no suco de laranja e nos preparados a quente que utilizaram o suco como única fonte de vitamina C, mostraram redução de aproximadamente 73\% no teor da mesma. Esta perda já é esperada, principalmente em relação a cocção, pois a natureza de seus ingredientes contribui para a degradação do ácido ascórbico, como o sal, açúcar e temperatura (FENNEMA, 1998). Estes resultados indicam instabilidade desta vitamina ao calor e possibilitam dimensionar a perda deste nutriente, permitindo que as previsões dietéticas sejam feitas de forma mais realista. O teor de ácido ascórbico em suco fresco de laranjas armazenadas sob refrigeração durante oito semanas variou entre 74,94mg\% e 51,55mg\%. Estes resultados indicam uma taxa de redução de aproximadamente de $0,92 \%$ ao dia do teor de ácido ascórbico. O teor médio do ácido ascórbico em suco in natura de laranja armazenado sob refrigeração durante sete dias foi de 49,62 mg\% com taxa de redução de 0,89\%/dia. Estas taxas foram menores quando comparada à obtida por Shaw e Moshanas (1991), onde estudaram o suco de laranja industrializado armazenado sob temperatura de $4,5^{\circ} \mathrm{C}$ e verificaram perda de 1,5 a $2 \%$ /dia e retenção média de ácido ascórbico de 88\% após 5 a 7 dias. Segundo Kabasakalis et al., (2000), não verificaram perda do ácido ascórbico em suco de laranja fresco armazenado sob refrigeração por dois dias.

Comparando-se os resultados obtidos nos dois experimentos, verifica-se que a taxa de redução do teor de AA é maior quando se compara o suco obtido de laranjas integras estocada sob refrigeração do que o suco mantido sob refrigeração por até sete dias. A maior retenção de AA observado no primeiro caso, quando comparado ao segundo caso, pode ser devido ao fato de o conteúdo interno das laranjas não estar em contato com a atmosfera evitando assim, perdas por oxidação. 
Os valores de acidez nas oito semanas analisadas não diferiram, tendo como menor média de 6,92 e maior média 8,03. Durante este período a média do ${ }^{\circ}$ Brix encontrado de 11,06. Os resultados encontrados estão de acordo com o que é estabelecido pela legislação brasileira vigente para o suco de laranja industrializado, que preconiza um teor mínimo de 7,0 para acidez e10,5iix.

Os valores do $\mathrm{pH}$ encontrados durante as oito semanas variaram de 4,19 a 4,52 com uma média de 4,37. A legislação brasileira não estabelece um valor mínimo de pH como identidade e qualidade para o suco de laranja industrializado. Segundo Neto e Faria (1999), valores característicos de frutas cítricas variam de 3,40 a 4,00.

\title{
4. Conclusão
}

Os sucos de laranja in natura são considerados uma das melhores fontes de vitamina C da dieta humana. A importância do coonhecimento do teor de ácido ascórbico nos alimentos bem como a extensão da sua perda durante o preparo, permite estimar a ingestão dietética correta deste nutriente planejando cardápios de forma a compensar as perdas esperadas.

O suco extraído de laranjas íntegras armazenado sob refrigeração $\left(4^{\circ} \mathrm{C}\right)$ não apresentou redução de ácido ascórbico. O suco in natura de laranja armazenado nas mesmas condições apresentou uma menor retenção do nutriente assim como em preparados quente, sendo escassos dados relativos à retenção de ácido ascórbico em temperaturas elevadas.

\begin{abstract}
It was done a comparative assessment of the vitamin $C$ retention (Ascorbic acid - AA) in fresh ofulb orange juice, storaged in refrigeration orange juice and cake with orange juice after it had been submitted to firing in a conventional oven. The AA content in juice wad of oranges that were refrigeratd for eight weeks has varied between 74, 94 and 51, $55 \mathrm{mg} \%$ with a reduction rate $0.92 \%$ in a day. In juice made of fresh oranges that were refrigerated for seven days, the AA content has varied between $49,62 \mathrm{mg} \%$, with an AA content reduction rate in about $0.89 \%$ in a day. The AA retaining was of $97.90 \%$ in the full oranges and in the refrigerated juice for seven dais it was of $50,38 \%$. The reduction of AA observed in the preparations the hot one was approximately $73 \%$, being necessary, in this case, to compensate the losses of this nutrient in the dietary planning.
\end{abstract}

Key-words: ascorbic acid, orange juice, thermal processing

\section{Referências}

BOBBIO, F. O.; BOBBIO, P. A. Vitaminas. Introdução à química de alimentos. São Paulo: Varela, cap. 5, p.163169. 1995.

BRASIL. Ministério da Agricultura. Instrução Normativa $n^{\circ} 1$, de 7 de Janeiro de 2000. Complementa padrões de identidade e qualidade para o suco de laranja. Diário Oficial da União da República Federativa do Brasil, Brasília, 10 de Janeiro de 2000.

CAMARGO, R. et al. Tecnologia de produtos agropecuários. São Paulo: Nobel, 1984. p. 310 
CHAMBERS, S. J.; LAMBERT, N.; PLUMB, G. W.; WILLIAMSON, G. Evaluation of the antioxidant properties of a methanolic extract from juice plus fruit and juice plus vegetable (dietary supplements). Food Chem, v.57, p.271- 2741996.

CORRÊA NETO, R.S.; FARIA, J.A.F. Fatores que influem na qualidade do suco de laranja. Ciência e Tecnologia de Alimentos, v.19, n. 1, p.153-160, 1999.

DAVIS, M. B.; AUSTIN, J.; PARTRIDGE, D. Vitamin C: its chemistry and biochemistry. Cambridge: Royal Society of Chemistry, 1991.

DOSUNMU, M. I. ; JOHNSON, E. C. Chemical evaluation of the nutritive value and changes in ascorbic acid contend during storange of the fruit of bitter kola (Garcinia kola). Food Chem, v.54, p.67-71, 1995.

FENNEMA, O. R. Química de los alimentos. 2 ed. Zaragoza: Acribia, 1993.1100 p.

GARDENER, P. T.; WHITE, T. A. C.; McPHAIL, D. B.; DUTHIE, G. G. The relative contributions of vitamin c, carotenoids and phenolics to the antioxidant potencial of fruit juices. Food Chem., v.68, p.471-474, 2000.

GAYET, J.P. Novo elo na cadeia. Agroanalysis. São Paulo, v.20, n. 2, fev. 1999.

GRANER, M.; FONSECA, H.; MONTENEGRO,T. L. N. Retenção de ácido ascórbico na elaboração de geléia de goiaba (Psidium guayava L.) a partir de polpa enlatada. O Solo, Piracicaba, n.1, p.61-63, 1977.

GOYLE, A.; OJHA, P. Effect of storage on vitamin, microbial load and sensory attributes of orange juice. Journal of Food Sciende and Technology - India, v. 35, n. 4, p.346-348, 1998.

INSTITUTO ADOLFO LUTZ. Normas Analíticas; Métodos Químicos e Físicos para Análise de Alimentos. $3^{\mathrm{a}}$ ed. São Paulo: 1995.

KABASKALIS, V.; SIOPIDOU, D.; MOSHATOU, E. Ascorbic acid content of commercial fruit juices and its rate loss upon storage. Food Chemistry, v. 70, p. 325-328, 2000.

KORDICK, J. O poder dos sucos. 8. ed. São Paulo: Ática, 1994. p.217.

REDDY, M.B.; LOVE, M. The impact of food processing on the nutritional quality of vitamins and minerals. Advances in Experimental Medicine and Biology, v. 459, p.99, 1999.

RUSCHEL, C.K.; CARVALHO, H.H.; SOUZA, R.B.; TONDO, E.C. Qualidade microbiológica e físico-química de sucos de laranja comercializados nas vias públicas de Porto Alegre - RS. Ciência e Tecnologia de Alimentos, v. 21, n.1, p. 94-97, 2001.

SADLER, G.D.; PARISH, N.E.; WICKER, L. Microbial, enzymatic and chemical changes during storage of fresh and processed orange juice. Journal of Food Science, v.57, n. 5, p.1187-1197, 1992.

SHAW, P.E.; MOSHONAS, M.G. Ascorbic acid retention in orange juice stored under simulated consumer home conditions. Journal of Food Science, v. 56, n. 3, p. 867-868, 1991.

VALENTE-MESQUITA, V.L.; LOPES, M.L.M.; SABINO, G.S.; SILVA, P.T.; ALVES, B.C. Teor de vitamina C em suco de cultivares de laranja (citrus sinensis) e em diferentes sucos industrializados. Nutrição Brasil, v.1, n.1, p.1-12, 2002.

WILLIANS, S.R. Fundamentos de Nutrição e Dietoterapia. 6 ed. Porto Alegre: Ed. Artes Médicas Sul LTDA, 1997.

\section{Dados do primeiro autor:}

Nome completo: Ana Paula de Faria Alves

Filiação institucional: Universidade Tecnológica Federal do Paraná, Campus Campo Mourão - PR.

Departamento: Coordenação do Curso Superior de Tecnologia de Alimentos

Função ou cargo ocupado: Tecnóloga em Alimentos

Endereço completo para correspondência: Rua Rolandia $n^{0} 209$. Conjunto Parigot de Souza Campo Mourão - Paraná. CEP: 87310-610.

Telefones para contato: (44) 9948-2045, (44) 3525-6985

e-mail: paulafarialves@gmail.com 